\title{
Light passage through the polymer film of liquid crystal droplets with modified boundary conditions
}

\author{
V.A. Loiko ${ }^{1}$, V.Ya. Zyryanov ${ }^{2}$, M.N. Krakhalev ${ }^{2}$, A.V. Konkolovich ${ }^{1}$, A.A. Miskevich ${ }^{1}$ \\ ${ }^{1}$ B.I. Stepanov Institute of Physics, National Academy of Sciences of Belarus, \\ 68, Nezalezhnastsi ave., Minsk, 220072 Belarus, e-mail: loiko@dragon.bas-net.by \\ ${ }^{2}$ L.V. Kirensky Institute of Physics, Siberian Branch of the Russian Academy of Sciences \\ Krasnoyarsk,660036, Russia
}

\begin{abstract}
A method to analyze coherent transmission coefficients and the small-angle light scattering structure of PDLC layer with homogeneous and inhomogeneous boundary conditions on the droplets surface is discussed. For PDLC films with inhomogeneous boundary conditions at the polymer - liquid crystal interface, asymmetry of the angular structure of light scattering with respect to the polar angle is revealed. Besides, experimental results on transmittance are presented.
\end{abstract}

Keywords: polymer dispersed liquid crystal, transmittance, scattering, light modulation.

Manuscript received 17.12.12; revised version received 22.02.13; accepted for publication 19.03.13; published online 25.06.13.

\section{Introduction}

Composite films of liquid crystal materials are the subject of active research, because they offer opportunities for creating display systems and optical information processing [1-5]. Using these films, it is possible to make multi-functional devices with controllable parameters, namely: intensity and phase modulators of light, polarizers and polarization converters of light, lenses, filters, reflectors, flexible displays, etc. [6-14].

A new method to control the structure of liquid crystal (LC) droplets in the polymer matrix by an electric field has been proposed recently [15]. It is based on modification of boundary conditions by the ionic surfactants. This method allows to reduce the value of the applied voltage in comparison with that for the ordinary polymer dispersed liquid crystal (PDLC) films.

Here, we consider a model to describe coherent transmission and the intensity of light scattered by monolayers of oriented spherical and ellipsoidal droplets with non-uniform surface adhesion. It is based on the amplitude-phase screen model, interference approximation of multiple wave scattering [9, 16, 17], and anomalous diffraction approximation to describe scattering of radiation by a single droplet. Numerical analysis of coherent transmission coefficients and scattered intensities were performed for monolayers of spherical droplets of nematic liquid crystals with inhomogeneous boundary conditions [18-20]. The internal structure of LC droplets was calculated using the relaxation method [21].

\section{Basic relations}

Let us consider a monolayer of oriented nonabsorbing LC droplets of ellipsoidal shape, dispersed in the nonabsorbing polymer matrix. Choose a laboratory coordinate system $(x, y, z)$, where the $x$ axis coincides with the normal to PDLC monolayer. All centers of the droplets are in the $(y, z)$ plane. Optical axes (directors) of the droplets coincide with their long axes in the absence of an external electric field and are oriented in the average along the $y$-axis. The layer is illuminated by a linearly polarized normal plane wave.

Consider components of the scattered wave parallel ( $v v$-component) and perpendicular ( $v h$-component) polarization with respect to the plane of polarization of 
the incident wave. These components correspond to parallel and crossed polarizer and analyzer geometries.

In the framework of the amplitude-phase screen model $[9,16], v v$ - and $v h$-components of the coherent transmittance ( $T_{c}^{v v}$ and $T_{c}^{v h}$, respectively) can be written as follows:

$$
\begin{aligned}
& T_{c}^{v v}=\left|T_{a}^{v v}\right|^{2}=1-\frac{4 \pi}{k^{2} \sigma} \eta \operatorname{Re} f_{v v}^{0}+\frac{4 \pi^{2}}{k^{4} \sigma^{2}} \eta^{2}\left|f_{v v}^{0}\right|^{2}, \\
& T_{c}^{v h}=\left|T_{a}^{v h}\right|^{2}=\frac{4 \pi^{2}}{k^{4} \sigma^{2}} \eta^{2}\left|f_{v h}^{0}\right|^{2} .
\end{aligned}
$$

Here, $\eta$ is the filling factor of the PDLC monolayer, which is numerically equal to the ratio of the section of all the LC droplets to the area they are distributed, $k=2 \pi n_{p} / \lambda$ is the wave vector, $n_{p}$ is the refractive index of the polymer matrix, $\lambda$ is the wavelength of incident light, $f_{v v, v h}^{0}$ are the $v v$ - and $v h$-components of the vector amplitude of the scattering function [22] in the forward direction $\left(\theta_{s}=0\right), \sigma$ is the cross-section of a droplet.

The coherent transmission coefficient for PDLC monolayer in the absence of polarization devices is the sum of (1) and (2), which can be written as:

$T_{c}^{p}=1-Q_{p} \eta+\frac{Q_{p}^{2} L_{T}}{2} \eta^{2}$.

Here, $Q_{p}$ is the extinction efficiency factor of linearly polarized light of a single droplet:

$Q_{p}=\frac{4 \pi}{k^{2} \sigma} \operatorname{Re} f_{v v}^{0}$,

$L_{T}=\frac{1}{2}\left(1+\frac{\operatorname{Im}^{2} f_{v v}^{0}}{\operatorname{Re}^{2} f_{v v}^{0}}\right)\left(1+\frac{\left|f_{v h}^{0}\right|^{2}}{\left|f_{v v}^{0}\right|^{2}}\right)$.

In the interference approximation [9, 16, 17], for the parallel $\left(I_{v v}\right)$ and perpendicular $\left(I_{v h}\right)$ plane of polarization of the incident radiation intensity components of incoherently (diffusely) scattered light, we obtain:

$$
\begin{aligned}
& I_{v v}\left(\theta_{s}, \varphi_{s}\right)=C \frac{\eta}{\sigma k^{2}}\left|f_{v v}\left(\theta_{s}, \varphi_{s}\right)\right|^{2} S\left(\theta_{s}\right), \\
& I_{v h}\left(\theta_{s}, \varphi_{s}\right)=C \frac{\eta}{\sigma k^{2}}\left|f_{v h}\left(\theta_{s}, \varphi_{s}\right)\right|^{2} S\left(\theta_{s}\right) .
\end{aligned}
$$

Here, $C=A E_{i}^{2} / R^{2}$ is the normalization constant, $A$ is the area of the test section of the sample, $E_{i}$ is the incident wave amplitude; $R$ is the distance from the origin to the point of observation, $f_{v v}\left(\theta_{s}, \varphi_{s}\right)$ and $f_{v h}\left(\theta_{s}, \varphi_{s}\right)$ are the $v v$ - and $v h$-components of the vector amplitude function scattering in the direction of the wave vector of the scattered wave $\mathbf{k}_{s}=\left(k \cos \theta_{s}, k \sin \theta_{s} \cos \varphi_{s}, k \sin \theta_{s} \sin \varphi_{s}\right)$, $S\left(\theta_{s}\right)$ is the structure factor of the monolayer [17], $\theta_{s}$ and $\varphi_{s}$ are the polar and azimuthal scattering angles. For a statistically isotropic ensemble of droplets with the shape of spheres or oblate spheroids, it does not depend on the azimuthal angle $\varphi_{s}$. At a low concentration of LC droplets, single scattering of light is implemented. In this case, the structure factor $S\left(\theta_{s}\right) \approx 1$ for all the values of the polar scattering angle. The difference of the structure factor from unity determines the degree of influence of interference effects on the angular structure of scattered light.

Using the elements of the amplitude scattering matrix $S_{j}(j=1,2,3,4)$, we have:

$$
\begin{aligned}
& f_{v v}\left(\theta_{s}, \varphi_{s}\right)=S_{2}\left(\theta_{s}, \varphi_{s}\right) \cos ^{2}\left(\alpha-\varphi_{s}\right)+ \\
& +S_{1}\left(\theta_{s}, \varphi_{s}\right) \sin ^{2}\left(\alpha-\varphi_{s}\right)+ \\
& +\frac{1}{2}\left(S_{3}\left(\theta_{s}, \varphi_{s}\right)+S_{4}\left(\theta_{s}, \varphi_{s}\right)\right) \sin 2\left(\alpha-\varphi_{s}\right), \\
& f_{v h}\left(\theta_{s}, \varphi_{s}\right)=S_{3}\left(\theta_{s}, \varphi_{s}\right) \sin ^{2}\left(\alpha-\varphi_{s}\right)- \\
& -S_{4}\left(\theta_{s}, \varphi_{s}\right) \cos ^{2}\left(\alpha-\varphi_{s}\right)+ \\
& +\frac{1}{2}\left(S_{2}\left(\theta_{s}, \varphi_{s}\right)-S_{1}\left(\theta_{s}, \varphi_{s}\right)\right) \sin 2\left(\alpha-\varphi_{s}\right) .
\end{aligned}
$$

For light scattered in the direction of the incident wave, the off-diagonal elements of the scattering matrix amplitudes are zero $\left(S_{3}=S_{4}=0\right)$. In this case

$$
\begin{aligned}
& f_{v v}^{0}=S_{2}^{0} \cos ^{2} \alpha+S_{1}^{0} \sin ^{2} \alpha, \\
& f_{v h}^{0}=\frac{1}{2}\left(S_{2}^{0}-S_{1}^{0}\right) \sin 2 \alpha .
\end{aligned}
$$

Here, $S_{2,1}^{0}$ are the diagonal elements of the amplitude matrix of scattering at zero scattering angle $\left(\theta_{s}=0\right)$.

In the anomalous diffraction (AD) approximation, the scattered field in the far zone is determined by diffraction on the amplitude-phase screen. The complex transmission matrix of the screen is determined via the amplitude scattering matrix elements [22]. It depends on the internal structure of the LC droplet orientation. The configuration of the director in the droplet with the given boundary conditions $[18,19,21]$ was found by solving the problem to minimize the bulk free energy density [23].

\section{Results}

Schematic presentation of the droplet cross-section and director orientation at the surface of the droplet with inhomogeneous boundary conditions is displayed in Fig. 1. The structure is symmetric with respect to the $y$ axis. There is normal and tangential orientation of liquid crystal molecules at the droplet-polymer interface. They are indicated in the figure by short lines that are normal and parallel to the surface, respectively. The parameter $W$ (see the figure) characterizes the fraction of the surface layer of the droplet with normal boundary conditions. $W$ is determined by the ratio of the surface 
segment height for the droplet with normal boundary conditions to the droplet diameter. The parameter values $W=0 \%$ and $100 \%$ correspond to fully homogeneous tangential and normal surface adhesion of LC molecules at the interface LC-polymer, respectively. When the parameter $W=0 \%$, there is a cylindrically symmetric bipolar configuration of the LC droplet with rigidly fixed poles. At $W=100 \%$, the internal structure of the LC droplets orientation is radially symmetric. Pay attention that Fig. 1 displays only a general director structure at the surface. In reality, transition from the tangential anchoring to the homeotropic one can be not sharp $[18,19]$.

The director configuration of the LC droplet is calculated in the Cartesian coordinate system by finding the distribution of directors of the elementary volumes of liquid crystal bulk in the droplet using the relaxation method of solving the bulk free energy density minimization problem [29]. The texture of the considered LC droplets with inhomogeneous boundary conditions, when the directions of analyzer and polarizer are crossed and $W=50 \%$, is illustrated by Fig. 2 .

The results of the coherent transmission $T_{c}^{p}(\rho)$ calculations show that, when illuminated with linearly polarized light with the polarization angle $\alpha=0$, the monolayer with the radial internal structure of droplets in a layer $(W=100 \%)$ has large values of the coefficient of the coherent transmittance in the range of the diffraction parameter $\rho>5(\rho=k a$, where $a$ is the radius of the droplets, $k$ is the wave number) as compared to the bipolar configuration of LC droplets $(W=0 \%)$. When a monolayer is illuminated with linearly polarized light with the polarization angle $\alpha=\pi / 2$, the situation is reversed: the values of $T_{c}^{p}(\rho)$ for the layer with tangential boundary conditions (and the bipolar configuration of LC droplets) are higher than the values $T_{c}^{p}(\rho)$ for the layer with normal boundary conditions (and the radial configuration of internal structure of droplets).

Electrooptical characteristics of the PDLC film with inhomogeneous surface adhesion on the liquid crystal droplet-polymer are illustrated in Fig. 3. The film was made with polymer PVA, liquid crystal 5CB, plasticizer Gl, and ionic surfactant CTAB. The droplet diameter is $3 \mu \mathrm{m}$, the film thickness is $75 \mu \mathrm{m}$, the bulk concentration is 0.094 . The wavelength $\lambda=0.658 \mu \mathrm{m}$. Ordinary refractive index of liquid crystal $n_{o}=1.531$, extraordinary refractive index of liquid crystal $n_{e}=1.717$, refractive index of polymer matrix $n_{p}=1.532$. Note that the results are obtained for the layer that consists of few monolayers. For one monolayer, the applied voltages will be lower.

It is well known that when we deal with a layer of droplets with homogeneous boundary conditions, the values of $I_{v v}\left(\theta_{s}\right)$ are identical for the same deviations in the polar scattering angle $\theta_{s}$ in different directions relative to the normal to the layer $\left(I_{v v}\left(\theta_{s}\right)=I_{v v}\left(-\theta_{s}\right)\right)$.

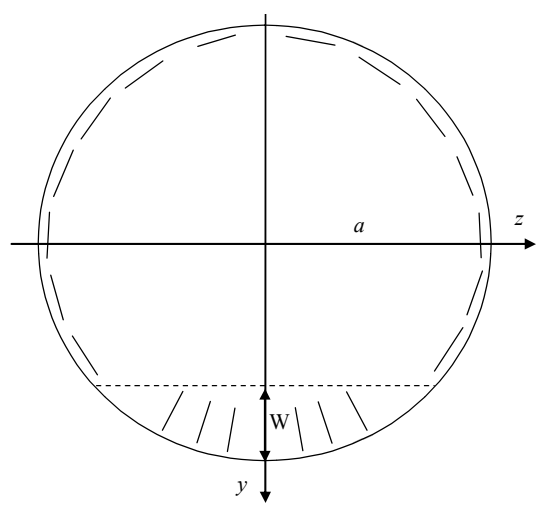

Fig. 1. Schematic presentation of the surface of a liquid crystal droplet with inhomogeneous boundary conditions.

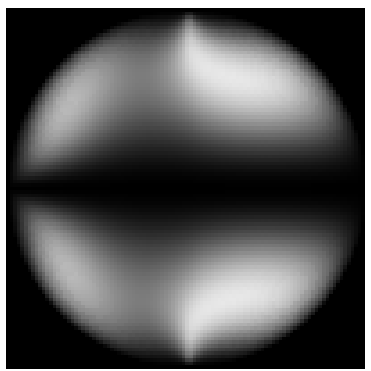

Fig. 2. Texture of LC droplet in crossed polarizers with inhomogeneous boundary conditions at $W=50 \%$. The $W$-value is determined by the ratio of the surface segment height for the droplet with normal boundary conditions to the droplet diameter, as it is shown in Fig. 1.
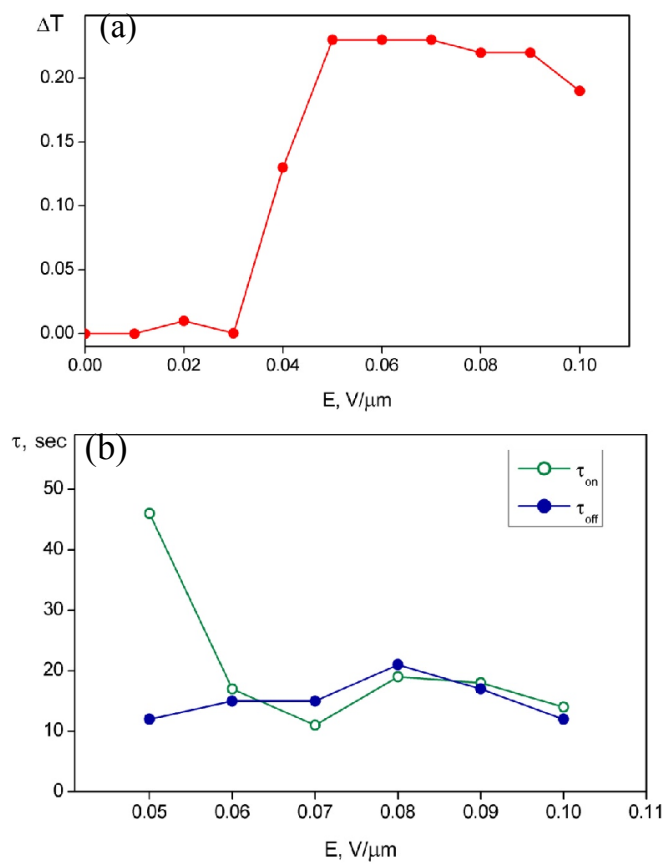

Fig. 3. Electrooptical characteristics of the PDLC film with inhomogeneous boundary conditions on the surface: coherent transmittance difference between on and off states $(a)$; response time $\tau_{o n}$ and relaxation time $\tau_{o f f}(b)$ vs the electric field strength $E$. 

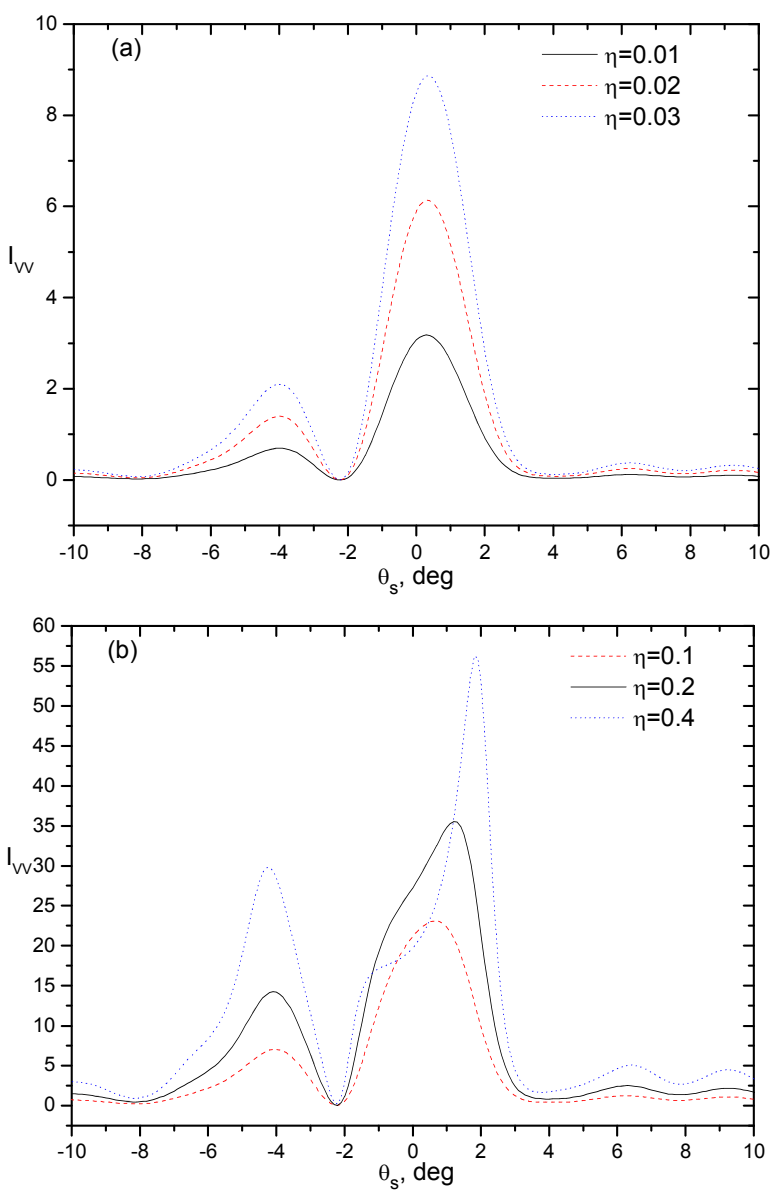

Fig. 4. Dependence of $I_{v v}$-component of the light intensity scattered by a monolayer of spherical LC droplets with inhomogeneous boundary conditions at different values of the filling factor $\eta=0.01,0.02,0.03(a), \eta=0.1,0.2,0.4(b)$. No field applied. $W=50 \%, \lambda=0.633 \mu \mathrm{m}, n_{o}=1.531, n_{e}=1.717$, $n_{p}=1.532, \eta=0.5, a=5 \mu \mathrm{m}, \alpha=0, \varphi_{s}=0$.

We have revealed that for a layer of droplets with inhomogeneous boundary conditions, such as "tangential-normal" ones, there is asymmetry of the angular structure of scattered light over the polar angle $\left(I_{v v}\left(\theta_{s}\right) \neq I_{v v}\left(-\theta_{s}\right)\right)$. The calculations show that it is the most pronounced when the parameter $W=50 \%$, i.e. with an equal share of the tangential and normal boundary conditions on the droplet surface. Analysis of the $v h$ component of the scattered light intensity shows that this component also possesses asymmetry in light scattering by the polar angle, but it is weaker in comparison with the $v$-component.

The influence of droplet concentration is illustrated in Fig. 4. At the considered parameters, one can see asymmetry in the angular structure for transmitted light for a wide range of concentrations. The angular structure of the concentrated layer has asymmetry determined by the specific scattering diagram of a single anisotropic droplet, and ordering the droplets due to their high concentration.

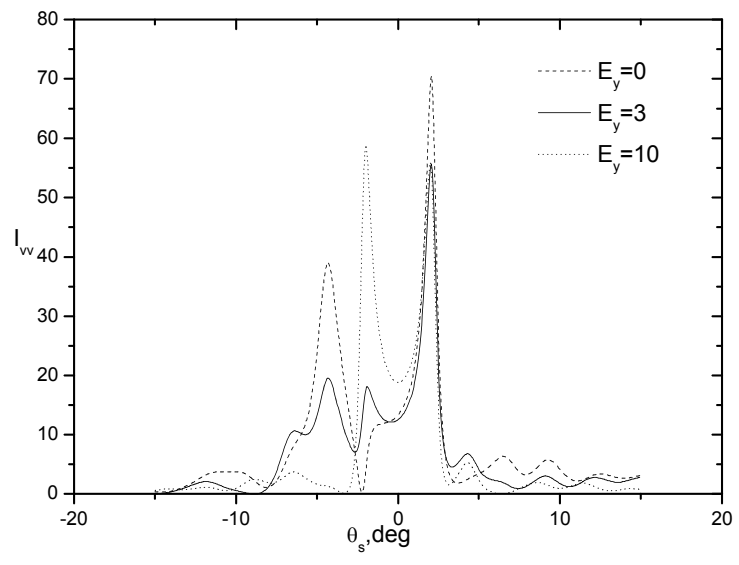

Fig. 5. Dependence of $I_{v v}$-component of the light intensity scattered by a monolayer of spherical LC droplets (in relative units, the normalization constant $C=1$ ) with inhomogeneous boundary conditions $(W=50 \%)$ as a function of the polar scattering angle $\theta_{s}$ with the value of the azimuthal scattering angle $\varphi_{s}=0$. The polarization angle $\alpha=0$, the refractive indices of LC $n_{o}=1.531, n_{e}=1.717$, the wavelength of incident light $\lambda=0.633 \mu \mathrm{m}$, the refractive index of polymer $n_{p}=1.53$, the droplets radius $a=5 \mu \mathrm{m}$, the filling factor of the monolayer $\eta=0.5$.

The influence of the electric field (normalized to the threshold value of the electric field) applied in-plane parallel to the optical axes of the droplets on the angular structure of light scattering at $W=50 \%$ is shown in Fig. 5. Asymmetry of the angular structure of scattering due to inhomogeneity of the boundary conditions has a pronounced character, up to the values of the normalized control field $E_{y}$ equal to 3, which corresponds to the ratio of the electrooptical response to the saturation one [20]. In a strong field, the angular structure of scattered light is more symmetric.

\section{Conclusion}

Light scattering by films containing droplets with homogeneous and inhomogeneous adhesion of liquid crystal molecules on the interface polymer - liquid crystal is investigated. The method for modeling and computing light propagation via a monolayer of liquidcrystal droplets dispersed in polymer matrix is developed.

The effect of asymmetry in the polar scattering angle for light scattered in the forward hemisphere for polymer films containing droplets with inhomogeneous boundary conditions has been revealed. This effect should be observed for light scattered in the backward hemisphere as well.

The experimental data on coherent (direct) transmittance, response time and relaxation time of the film containing droplets with inhomogeneous boundary conditions under the action of the electric field are displayed. 
The obtained results can be used to analyze transmittance, angular structure, and polarization characteristics of light scattered by a monolayer of liquid crystal droplets with homogeneous and inhomogeneous boundary conditions.

\section{Acknowledgements}

This work was performed under the aegis of the Integration Project and supported by the Belarusian Republican Foundation for Fundamental Research, Project No. F12SO-007.

\section{References}

1. F. Simoni, Nonlinear Properties of Liquid Crystals and Polymer Dispersed Liquid Crystals. World Scientific, Singapore-New Jersey-London-Hong Kong, 1997.

2. P.S. Drzaik, Liquid Crystal Dispersions. World Scientific, Singapore-New Jersey-London-Hong Kong, 1994.

3. M. Kleman, O.D. Lavrentovich, Soft Matter Physics. New York, Springer, 2002.

4. O. Yaroshchuk, F. Elouali, U. Maschke, Control of phase separation and morphology of thiolene based PDLCs by curing light intensity // Opt. Mater. 32, p. $982-989$ (2010).

5. V.G. Chigrinov, Liquid Crystal Devices: Physics and Application. Boston-London, 1999.

6. V.Ya. Zyryanov, S.L. Smorgon, V.F. Shabanov, Elongated films of polymer-dispersed liquid crystals as scattering polarizers // Molecular Eng. 1, p. 305-310 (1992).

7. F. Bloisi, L. Vicari, and F. Simoni, Optical phase shift of polymer-dispersed liquid crystals // Phys. Rev. E, 48, p. $432-438$ (1993).

8. H. Ren, Y.-H. Lin, Y.-H. Fan, and S.T. Wu, Tunable-focus microlens arrays using nanosized polymer-dispersed liquid crystal droplets // Opt. Communs. 247, p. 101-106 (2005).

9. V.A. Loiko, A.V. Konkolovich, Interference effect of coherent transmittance quenching: theoretical study of optical modulation by surface ferroelectric liquid crystal droplets // J. Phys. D: Appl. Phys. 33, p. 2201-2211 (2000).

10. V.A. Loiko, A.V. Konkolovich, A.A. Miskevich, Using the Wentzel-Kramers-Brillouin method to analyze the transmission and reflection of light by a layer of liquid-crystal drops dispersed in a polymeric matrix // J. Opt. Technol. 78, p. 48-54 (2011).

11. V.P. Dick and V.A. Loiko, Optical phase shift by polymer dispersed liquid crystal films with findroplets // J. Phys. D, 37, p. 1834-1840 (2004).
12. V.A. Loiko, A.V. Konkolovich, Focusing of light by polymer-dispersed liquid-crystal films with nanosized droplets // JETP, 103(6), p. 935-943 (2006).

13. P.G. Lisinetskaya, A.V. Konkolovich, A.V. Loiko, Polarization properties of polymer-dispersed liquidcrystal film with small nematic droplets // Appl. Opt. 48(17), p. 3144-3153 (2009).

14. L.O. Dolgov and O.V. Yaroshchuk, Light scattering liquid-crystal composites with reduced off-axis haze // J. SID, 14, p. 657-662 (2006).

15. O.O. Prishchepa, V.Ya. Zyryanov, A.P. Gardymova, V.F. Shabanov, Optical textures and orientational structures of nematic and cholesteric droplets with heterogeneous boundary conditions // Mol. Cryst. and Liquid Cryst. 489, p. 84-93 (2008).

16. V.A. Loiko, A.V. Konkolovich, A.A. Miskevich, U. Maschke, Phase modulation of light by a composite film with fine ferroelectric liquid crystal droplets: theoretical treatment // Ferroelectrics, 344, p. 111-115 (2006).

17. V.A. Loiko, U. Maschke, V.Ya. Zyryanov, A.V. Konkolovich, A.A. Miskevich, Small-angle light scattering from polymer-dispersed liquid-crystal films // J. Experim. and Theor. Phys. 107(4), p. 692-698 (2008).

18. V.Ya. Zyryanov, M.N. Krakhalev, O.O. Prishchepa, Texture transformation in nematic droplets caused by ionic modification of boundary conditions // Mol. Cryst. and Liquid Cryst. 489, p. 273-279 (2008).

19. V.Ya. Zyryanov, M.N. Krakhalev, O.O. Prishchepa, A.V. Shabanov, Orientational structure transformations caused by the electric-fieldinduced ionic modification of the interface in nematic droplets // JETP Lett. 86, p. 383-388 (2007).

20. M.N. Krakhalev, V.A. Loiko, V.Ya. Zyryanov, Electro-optical characteristics of polymer-dispersed liquid crystal film controlled by ionic-surfactant method // Technical Physics Letters, 37(1), p. 34$36(2011)$.

21. O.O. Prishchepa, A.V. Shabanov, V.Ya. Zyryanov, A.M. Parshin, V.G. Nazarov, Friedericksz threshold field in bipolar nematic droplets with strong surface anchoring // JETP Lett. 84(11), p. 607-612 (2007).

22. H.C. van de Hulst, Light Scattering by Small Particles, Dover Publ. Inc., New York, 1957.

23. R. Ondris-Crawford, E.P. Boyko, B.G. Wagner, J.H. Erdmann, S. Zumer, J.W. Doane, Microscope textures of nematic droplets in polymer dispersed liquid crystal // J Appl. Phys. 69, p. 6380-6386 (1991). 